\title{
EXPERIMENTAL AND NUMERICAL INVESTIGATION ON A TROPOSKEIN VERTICAL AXIS WIND TURBINE
}

\author{
N. Franchina ${ }^{1}$, G. Persico ${ }^{2}$, M. Savini ${ }^{1}$ \\ ${ }^{1}$ Dipartimento di Ingegneria e scienze applicate, Università degli Studi di Bergamo, Viale \\ Marconi 5 - 24044 Dalmine (BG), Italy \\ ${ }^{2}$ Dipartimento di Energia, Politecnico di Milano, via Lambruschini 4 - 20156, Milano
}

\begin{abstract}
The Vertical Axis Wind Turbine (VAWT) is a promising technology for energy harvesting in complex environments, as urban sites and floating offshore installations, which experience aerodynamic complexity due to the platform motion. In this paper, we present a numerical investigation into the aerodynamics and performance of a troposkein VAWT, supported by a systematic experimental assessment on the wind-tunnel model of a suitable rotor. The flow in the rotor equatorial plane is first investigated over the entire operational curve of the turbine, applying a validated computational model. A fully three-dimensional analysis is then carried out for one specific tip speed ratio, in order to investigate the spanwise evolution of the rotor aerodynamics and the system of vortices generated by the peculiar shape of the rotor. It is shown that these three-dimensional motions influence the flow and the evolution of the wake also in the midspan section. The observed flow features are also discussed in the perspective of a comparison with the aerodynamics of a H-shape turbine featuring the same swept area, blade number, and blade profile.
\end{abstract}

\section{Nomenclature}

\begin{tabular}{|c|c|c|c|}
\hline $\mathrm{c}$ & blade chord $[m]$ & $\mathrm{P}$ & power $[W]$ \\
\hline $\mathrm{k}$ & turbulent kinetic energy $\left[\mathrm{m}^{2} / \mathrm{s}^{2}\right]$ & $\mathrm{R}$ & turbine radius $[m]$ \\
\hline $\mathrm{n}$ & rotational speed $[\mathrm{rad} / \mathrm{s}]$ & \multicolumn{2}{|c|}{$\begin{array}{l}\text { URANS Unsteady Reynolds Averaged Navier- } \\
\text { Stokes }\end{array}$} \\
\hline$C_{M}$ & $\begin{array}{l}\text { moment coefficient } \\
C_{N}=\mathrm{M} /\left(1 / 2 \Omega_{0} \mathrm{~V}^{2} \mathrm{AR}\right)[-]\end{array}$ & $R e$ & Reynolds number $[-]$ \\
\hline \multirow[b]{2}{*}{$C_{P}$} & & SST & Shear Stress Transport \\
\hline & $\begin{array}{l}\text { power coefficient } \\
C_{P}=C_{M} T S R[-]\end{array}$ & TSR & Tip Speed Ratio \\
\hline A & turbine frontal area $\left[\mathrm{m}^{2}\right]$ & $T u$ & turbulence intensity $\mathrm{Tu}=\sqrt{2 k / 3} / \mathrm{V}_{\infty}[-]$ \\
\hline $\mathrm{D}$ & turhine diameter $[\mathrm{m}] \mathrm{drag}[\mathrm{N}]$ & $\mathrm{U}$ & peripheral velocity $[\mathrm{m} / \mathrm{s}]$, uncertainty [-] \\
\hline D & curvine urameter $[m]$, urag $[\mathrm{v}]$ & $\mathrm{V}$ & absolute velocity $[\mathrm{m} / \mathrm{s}]$ \\
\hline $\mathrm{L}$ & lift $[N]$, blade length $[m]$ & \multicolumn{2}{|c|}{ VAWT vertical-axis wind turbine } \\
\hline $\mathrm{H}$ & turbine span $[m]$ & $\mathrm{W}$ & relative velocity $[\mathrm{m} / \mathrm{s}]$ \\
\hline M & moment $[N m]$ & $\alpha$ & incidence angle $[\mathrm{deg}]$ \\
\hline
\end{tabular}




$\begin{array}{llll}\omega & \text { vorticity }[1 / s] & \Delta t & \text { time step }[s] \\ \rho & \text { density }\left[K g / m^{3}\right] & \Delta x & \text { spatial discretization parameter }[m] \\ \sigma & \text { solidity } \sigma=\mathrm{NcL} / \mathrm{A}[-] & \infty & \text { freestream } \\ \vartheta & \text { azimuthal angle }[\mathrm{deg}] & e q & \text { equatorial }\end{array}$

\section{Introduction}

Although the history of the Vertical Axis Wind Turbine (VAWT) dates back to ancient times and the Darrieus concept outperformed any other device available at the time of its presentation, the VAWT eventually lost the competition (among modern lift-driven wind turbines) against the Horizontal Axis Wind Turbine (HAWT), mainly because of its lower efficiency.

An excellent picture of the VAWT technology at the beginning of this Century is depicted in the book of Paraschivoiu [17]. This review clarifies the difficulties involved in the development of VAWT technology, that lie in the aerodynamic complexity and in the structural fatigue loads of a machine which is, of necessity, unsteady. However, the text also highlights some advantageous aspects with respect to HAWTs: insensitivity to yaw angle, low sensitivity to gust and flow skewness, lower noise (due to its lower optimal tip-speed ratio), the possibility to mount gearbox, electric generator, and other equipments at the ground level. In the last decade, the development of VAWT technology has seen a significant acceleration. Recent works have enriched the experimental database on prototypes at laboratory scale $[9,22,13,8,3]$, have improved the modeling techniques $[21,1,4,19,10]$, and have investigated issues related to the machine operation $[23,5,15,11]$. This has improved the VAWT concept, making it progressively more competitive.

The above mentioned considerations have led to rethink the VAWT as a promising technology for the efficient energy harvesting in non-conventional environments, especially for small-scale urban and/or distributed installations. The same arguments, however, apply also in another, extremely relevant scope, i.e. Floating Offshore Wind Turbines (FOWT), which experience a significant flow complexity due to the movement of the platform. FOWTs operate under time-periodic skewness and yaw conditions and, in case of HAWTs, have to cope with large overturning moments due to heavier components of the turbine, such as the drivetrain and the generator which have to be placed high above the sea level [16]. In such a context, VAWTs may be better suited, as heavy components would all sit at the base of the turbine, and the rotor aerodynamics is less sensitive to wind skew and yaw with respect to HAWTs. VAWTs could thus potentially enhance the turbine performance and, at the same time, reduce the cost of FOWTs installation and maintenance, owing to the lack of gearboxes, high-speed shafts, yaw regulation systems and nacelles which are all potentially subject to faults.

An example of VAWT conceived for FOWT applications is the DeepWind turbine, proposed in the frame of a EU-FP7 research program [24]. As demonstrated in the DeepWind Project, the scaling of VAWT rotors to large dimension, necessary to reduce the levelled cost of electricity, makes the troposkein rotor architecture the unique design suitable for FOWT applications. However, most of the recent studies cited above are focused on simpler straight-blade, or $\mathrm{H}$ shape, rotors, and very few research papers deal with the detailed aerodynamics of troposkein rotors (even though this shape was originally proposed in the Darrieus patent and is extensively discussed, also for its structural implications, in many texts). Results of experimental studies on laboratory models of troposkein VAWTs are presented in $[2,18,3]$, and only few computational studies are available, mostly carried out with simplified aerodynamic models (see [20]) or with 

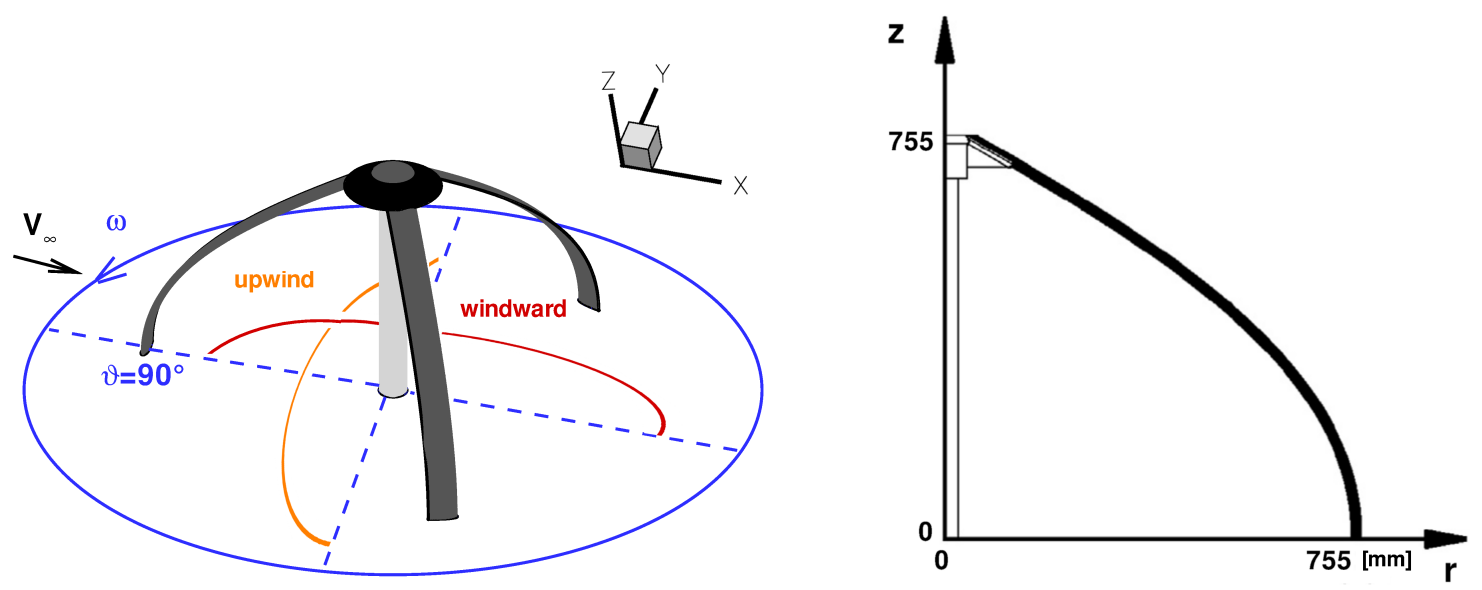

Figure 1: Troposkein VAWT - blade radial profile $\mathrm{r}(\mathrm{z})$

CFD $[6,7]$. The motivation for the still limited research on this class of machines has to be found in the severe complexity of the problem, which forces to resort to fully 3D and unsteady simulations and, in general, to High Performance Computing (HPC).

A deep understanding of the complex fluid dynamic field around VAWTs in skewed flows, as well an accurate prediction of their performance, require the use of high-resolution prediction CFD tools. To the best of the authors knowledge, few works devoted to fully 3D numerical investigation are available, e.g. [15, 12, 23, 19]. In this paper we use both numerical and experimental results to investigate a troposkein VAWT, considering a small-scale wind-tunnel model of a rotor theoretically suitable for upscaling to FOWT applications. By means of a systematic comparison among wind-tunnel experiments, two-dimensional and the fully three-dimensional CFD simulations of the turbine flow field, we highlight the three-dimensional character of the troposkein rotor and, by virtue of capitalizing the previous experiences on a $\mathrm{H}$-shape turbine, we establish a first comparison between different VAWT rotor architectures. The H-VAWT turbine used for comparison employs the same airfoil profile and chord, the same number of blades, and sweeps the same area.

The paper is structured as follows. At first the flow in the equatorial plane of the turbine is investigated applying a previously validated 2D CFD model over the full turbine operating range, and a first experimental comparison is proposed. Then a 3D CFD analysis is presented and discussed for one relevant operating condition in order to investigate the evolution of rotor aerodynamics along the axis of the machine, away from midspan, and the fully $3 \mathrm{D}$ nature of the flow field. Finally a brief analysis of the unsteady vortical motions inside the rotor and in the downstream wake concludes the paper.

\section{Turbine configuration, experimental and numerical set-up}

Figure 1 shows the upper half of the turbine and the coordinate system; the blade radial profile $r(z)$, set at mid-chord of the airfoil, can be found in [3]. Note that a typo occurred in Table 7 of the quoted reference and the axial coordinate of points $n .99$ and n.100 must be corrected, respectively, to 107 and 75. Starting from $\vartheta=0^{\circ}$, the first and second quarters are the upwind side, the third and fourth the downwind one; similarly the fourth and first quarters are the windward part of the revolution, the second and third the leerward one, as the blade advance toward the wind in the formers and retreat from it in the latters. 

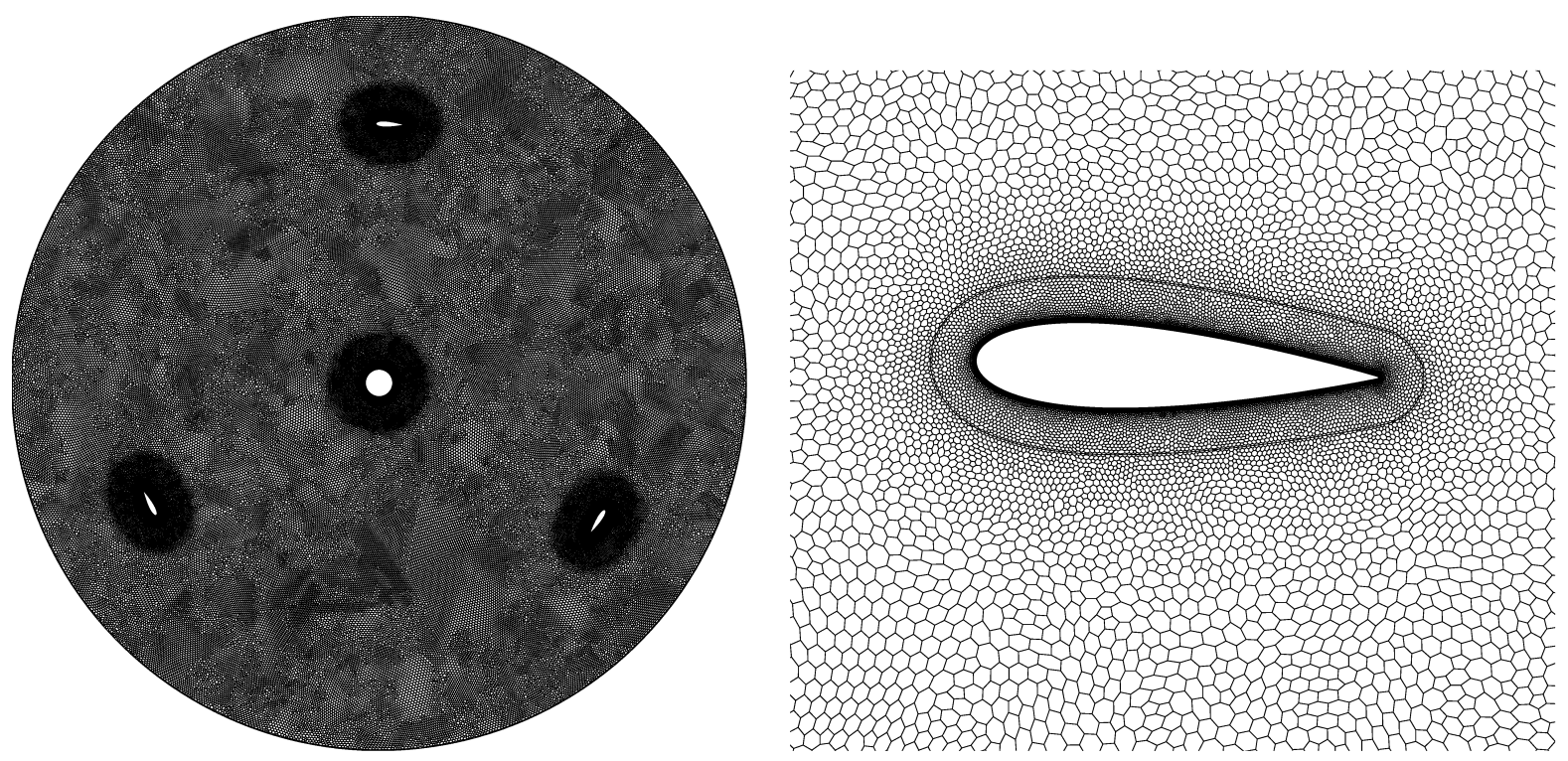

Figure 2: Two-dimensional grid

Note that the NACA0021 profile is orthogonal to the troposkein curve $r(z)$ and not to the axis of rotation. Therefore, in all but the midspan section, the profile cut in a plane normal to the axis of rotation has a maximum thickness greater than $21 \%$ of the chord. It is an open question which radius should be taken as representative of the mean fluidynamic behaviour of the turbine; the simple average radius is clearly inappropriate and both $\bar{R}_{2}$ and $\bar{R}_{3}$, defined using the square and cubic averages, can be used. We prefer the root mean square

$$
\bar{R}_{2}=\sqrt{\frac{1}{H} \int_{0}^{H} r^{2}(z)} d z,
$$

since aerodynamic forces and moments are roughly proportional to $r^{2}$, at least not far from midspan, where most of flow rate is passing and the local TSR is much greater than one (and hence the relative velocity is close to the peripheral one). In our turbine $\bar{R}_{2}$ is located at three quarters of the semi-span $H^{*}$ toward midspan, where the troposkein slope is 43 degrees: the maximum thickness in this point corresponds therefore to $31 \%$ of the chord.

The Troposkein turbine was investigated experimentally in the large-scale wind tunnel of the Politecnico di Milano. It was tested in both confined and unconfined mode; in this work only the unconfined (free-jet) conditions are considered, for which the available correlations [14] estimate a negligible blockage (below 2.5\%). Measurements performed included the turbine axial and lateral thrust as well as the shaft torque and, ultimately, the turbine power. Velocity and turbulence measurements in the turbine wake were obtained by means of miniature hot-wire anemometers. Full details on the turbine geometry and on the measurement system, as well as performance data, are reported in [3]; for a detailed analysis of the wake of this troposkein rotor the reader is referred to [18]. In the present work, non-dimensional performance data are provided with their specific uncertainty, represented as error bar. The uncertainty in the velocity measurements was estimated in $2 \%$ of the local value.

Both two-dimensional and three-dimensional simulations are hereafter presented and discussed; the former are carried out on the equatorial plane of the turbine while the latter on half 


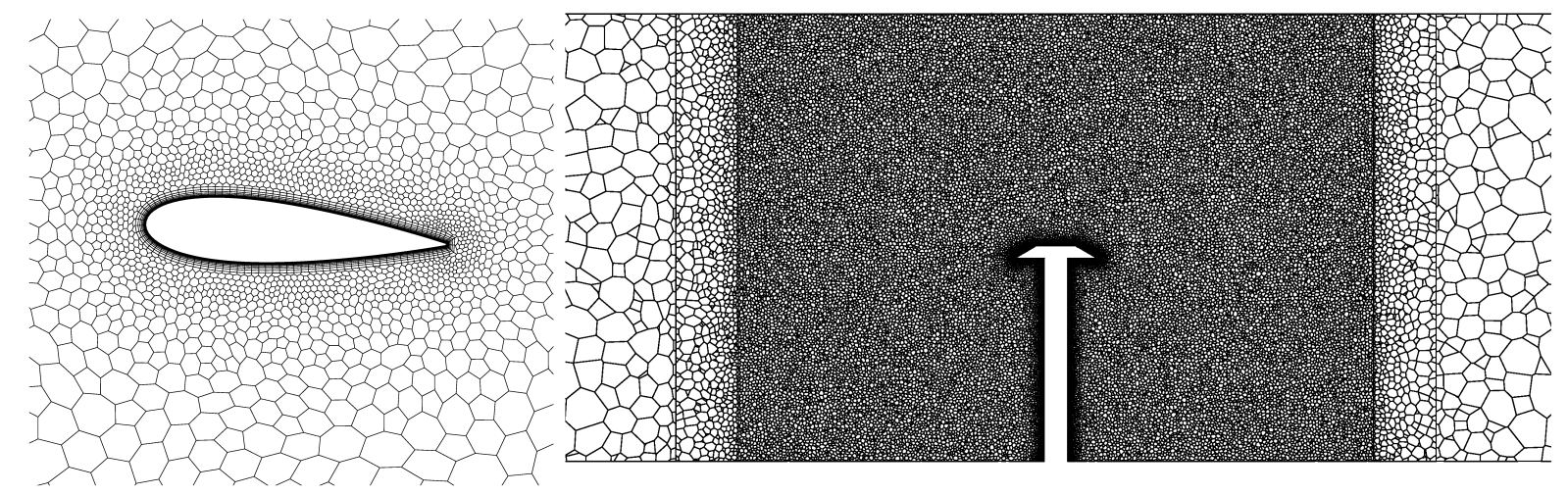

Figure 3: Three-dimensional grid

the turbine model. Relying upon [10], where the authors performed a grid convergence study related to both the near-wall region and the global resolution level, an unstructured 2D mesh with nearly 200.000 cells of general shape (including polygonals) is here adopted, extending 4.5 turbine diameters upstream the axis of rotation and 3 diameters both laterally and downstream. The minimum spacing normal to the airfoil surfaces is $2 \cdot 10^{-4}$ times the blade chord, sufficient in all cases here reported to obtain everywhere a $y^{+}$value lower than one. Total number of cells and their tight spacing exceed the normal requirements of such kind of a computation. Figure 2 illustrates a global view of the mesh, in particular the circled rotating internal domain, as well as a close-up near the blade solid surface.

Keeping the same characteristic dimensions in the third dimension would have led to a computational grid of roughly 45 millions elements, far beyond what is affordable by the computational resources at our disposal and maybe even necessary. Therefore we built a coarser three-dimensional mesh of approximately 10.7 millions elements, number which proved to be satisfactory in the preceding tests reported in $[10,11]$. This may raise doubts about the true grid-independency of the 3D results and therefore we used also another two-dimensional grid, obtained extracting the plane of symmetry of the 3D domain. It consists of 50 thousands cells and will be used for the sole purpose of grid convergence study. It will be termed in the following the "coarse" mesh (2Dc), as opposed to the "fine" one (2Df). The substantial equivalence of the results obtained with the two different 2D grids, as discussed later, allows to infer that the 3D mesh is fine enough to catch all relevant phenomena.

The 3D domain extends up to $z=D_{e q}$ and Figure 3 gives an idea of cell spacing both near the airfoils (in the equatorial plane) and in the axial direction $z$.

The code used to solve the URANS equations is Siemens' STAR-CCM+, with the incompressible constant-properties segregated flow solver, employing the $k-\omega$ SST turbulence model and a second-order accurate discretization both in space and time.

In every test run, more than 15-20 turbine revolutions were needed to establish a stable flow field. The integral of the torque collected at the shaft over one revolution was recorded and the last ten integral values continuously analyzed to check the achievement of a satisfactory periodicity. At the highest TSR investigated equal to (4.0) the torque rms was of $7 \cdot 10^{-4}$ its mean value, at the lowest TSR (i.e. 2.0) $1 \cdot 10^{-2}$.

Boundary conditions were imposed as it follows: velocity fixed at the inlet boundary, altogether with a turbulence intensity of $1 \%$ and a turbulent viscosity ratio equal to 1 ; these values have 

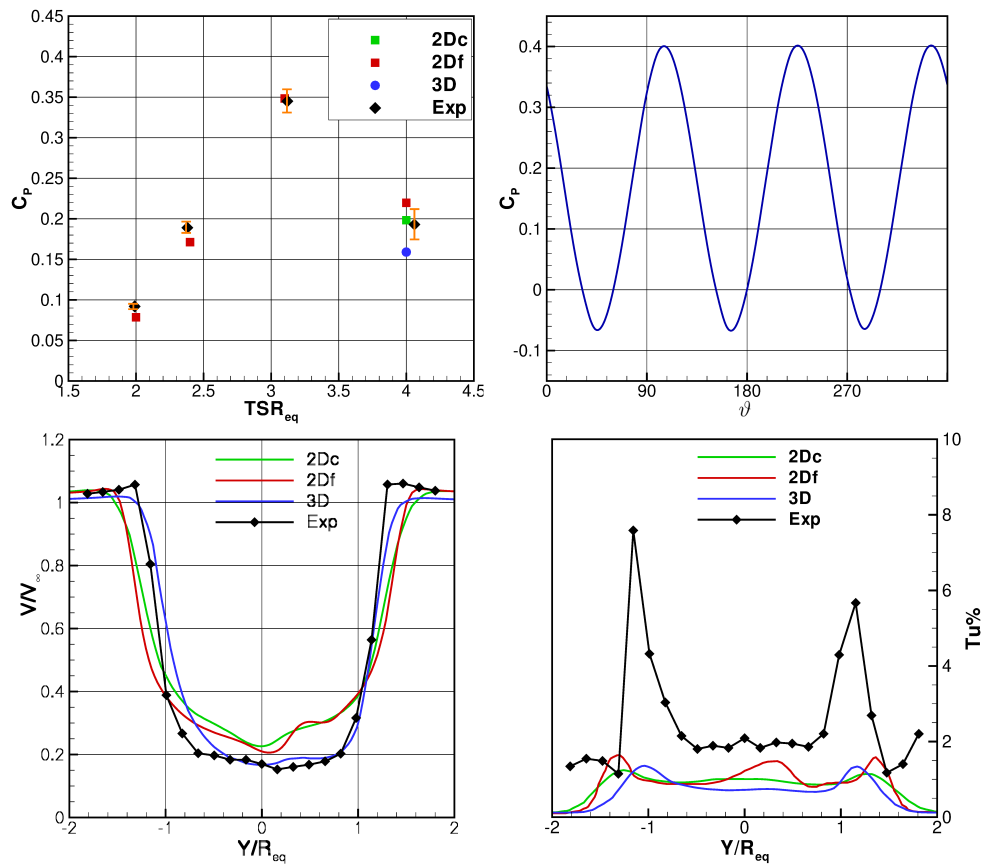

Figure 4: Performance and wake profile: Power coefficient versus TSR/top left) and versus azimuthal angle/top right (3D results at $\mathrm{TSR}=4.0$ ), Streamwise velocity (bottom left) and turbulence intensity (bottom right) at $x / R_{e q}=1.7$ for TSR $=4.0$.

been selected according to the experimental turbulence level considered. Notice that these values also meet the values typically adopted by the turbulence model employed. At lateral and downstream boundaries, static pressure is prescribed while the no-slip condition is imposed at solid surfaces.

\section{2D-3D comparison and experimental assessment}

2D computations were run at four different $\operatorname{TSR}_{e q}$ (namely 2,2.4,3.1,4), sweeping the whole operating range of the turbine. We observe that these results resembles the ones obtained for the $\mathrm{H}$-shaped VAWT investigated in [3,11], which shares blade geometry, blade number, turbine swept area, both in terms of $C_{P}$ (see top left part of Figure 4) and of flow field relevant features. The Figure also illustrates the $C_{P}=f(\vartheta)$ distribution of the wind turbine at TSR=4.0 (3D results).

One of the main difference between the two machines architectures is the right shift of performance curve toward greater TSR of the troposkein one (see [3]) which is consistent with the related lower solitidy in the equatorial plane ( 0.17 instead of 0.25 for the H-shaped one). Observe that in 2D, it is usual to express the solidity as $\sigma=N c / D$. However, on the basis of the definition commonly used (reported in the nomeclature) the solidity of the 3D troposkein turbine involves the blade length and it is greater (0.38) than that of the H-shaped one. Therefore, in comparison to this latter, the effect of solidity would go in the opposite direction if resorting alternatively to $2 \mathrm{D}$ or $3 \mathrm{D}$ models of the machine. Comparison with experiments sheds light on this aspects; for the moment, we note that $\sigma$ should not depend on the blade length (as done in axial turbomachinery). It is also worth to mention that the optimum $C_{p}$ of the troposkein VAWT is somewhat greater (about 0.34 instead of 0.29), as found in the experiments. 
Figure 5 gives evidence of the different aerodynamic behaviour and wake development at low TSR: the great angles of attack experienced by the blade lead to detachment of vortices in the leeward side and to less extraction of work from the wind stream: the wake is thinner and its velocity deficit smaller.

Looking at the performance results, an unexpected very good agreement is found between the 2D simulations and the measured power coefficients. The fluid dynamics of a Darrieus-type VAWT is, in fact, fully three-dimensional; both TSR, solidity, and frontal area (i.e. blockage) vary in the axial direction; moreover, for the tested turbine, also the shape of the blade section in the rotational plane changes dramatically from midspan to tip: how can a 2D simulation at midspan be representative of the behaviour of the actual turbine?. Leaving this question open for the moment, an hasty inference may lead to expect the same level of agreement in terms of local data obtained from the experimental wake traverses at midspan. This is not at all the case, as shown in the bottom part of Figure 4, where measurements at TSR=4 are compared with the outcomes of 2D and 3D computations.

The velocity profiles obtained with the two bi-dimensional simulations, on the coarse and fine meshes, are very much the same (as their power coefficients) but differ significantly from the experiments. In particular, the wake is larger than the actual one and it is characterized by a smaller velocity deficit. These differences are related to the lower blockage effect exerted by the troposkein turbine, effect mitigated by the third dimension, and to its better extraction of the wind kinetic energy. It is reasonable to state that here the midspan section of the VAWT works better than if it were that of an $\mathrm{H}$-shaped machine. By the way, from the similitude of the $2 \mathrm{D}$ results obtained with different grids, we can conclude that the coarseness of the $3 \mathrm{D}$ grid does not impair significantly the accuracy of the results, at least at this TSR. For what concerns the turbulence profiles, it must be kept in mind that the computed Tu ought to be smaller than the measured one. This issue has already deeply analyzed and discussed in [11], in particular as therein reported, in the experiments, only the unresolved velocity fluctuation is measured (i.e. the streamwise component) and any disturbance (not related to the rotational frequency) is considered "turbulence". Instead, the CFD results relies on a turbulence model based upon the hypothesis of isotropic normal Reynolds stresses. That said, it is evident also in this graph how the peaks of turbulence intensity are slightly displaced outwards in the 2D case, indicating a wider wake. Moreover, there is an increase of the $T u$ level near $Y / R_{e q}=0$, due to the vortex street behind the mast, not visible in the experiments. The three-dimensional simulation capture very well the velocity profile, as well as the correct, from a qualitative point of view, turbulence transverse distribution.

Let's now come back to discuss why there is such a good agreement between the $2 \mathrm{D} C_{P}$ curve and the real one, opposite to what experienced in an H-shaped VAWT, where passing from a $2 \mathrm{D}$ to a $3 \mathrm{D}$ turbine representation always leads to a decrease of the performance, more pronounced at high TSRs (see e.g. [11]). The first observation is that, at $\mathrm{TSR}_{e q}=4$, the mean TSR (based on $\bar{R}_{2}$ ) is 2.9 , that is exactly in the region of predicted maximum $C_{P}$ for the previously studied H-VAWT. Therefore this fact should play in favour of a better performance for the fully 3D turbine model. Another favourable item is the reduced mean blockage, as near the midspan section, the flow is allowed to escape in the axial direction, toward the outer turbine sections, and is not forced to skip laterally. We will see in the next section how this is clearly reflected in a very different wake spreading angle of the 3D simulation. On the other hand, moving toward the tip sections, the solidity gets greater and greater, intensifying the friction on wet surfaces and obstructing the flow passage. At the same time, as previoulsy mentioned, the airfoil profile normal to the axis of revolution gets thicker and, as well known, sees its aero- 

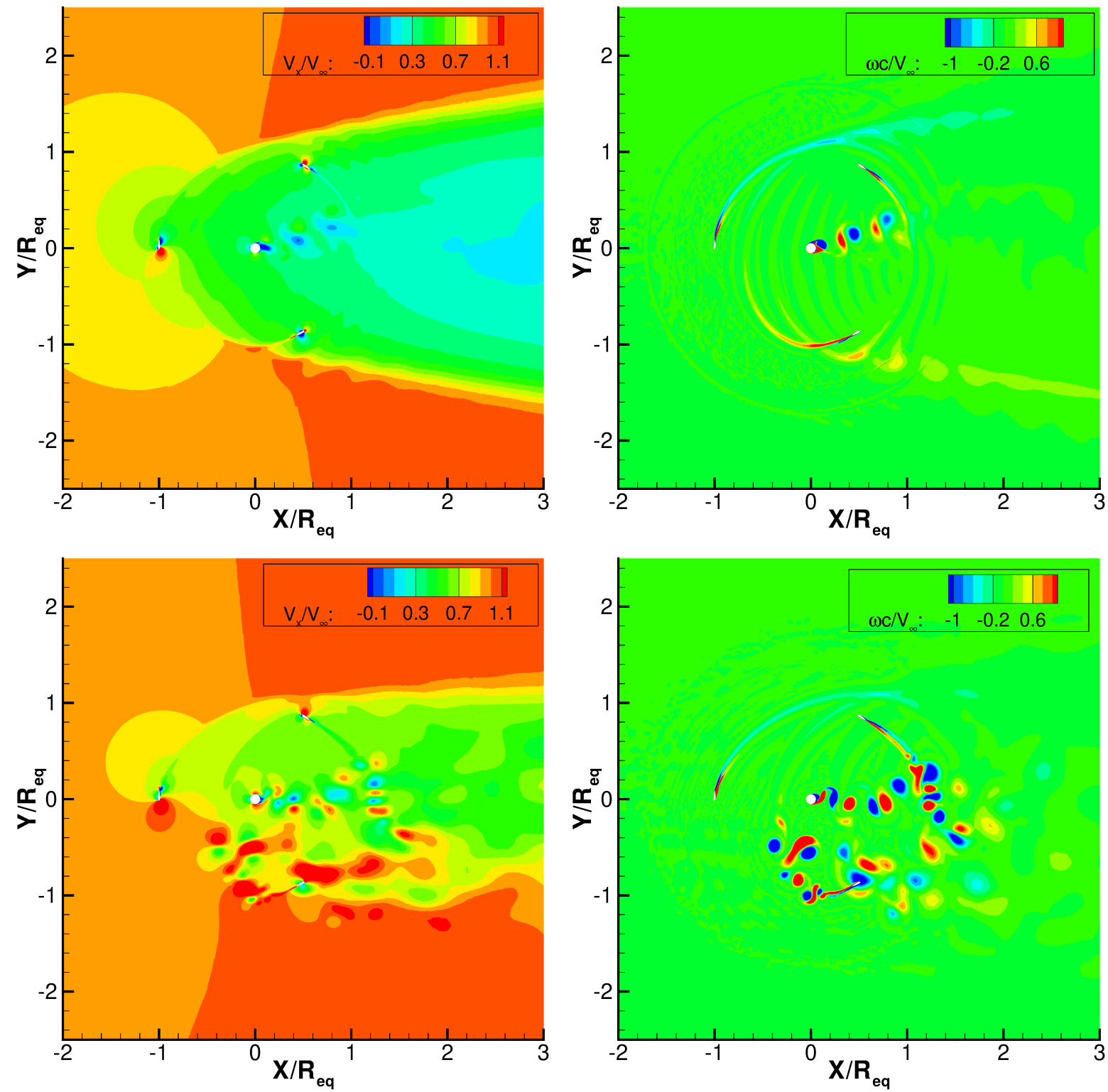

Figure 5: $\mathrm{TSR}=4$ (top) and $\mathrm{TSR}=2.4$ (bottom): Non dimensional velocity (left) and vorticity (right) fields when $\vartheta=90^{\circ}$. 
dynamic efficiency reduced at small angles of attack (especially at low $R e$ ). Last but not least, secondary vortices are expected to form under the action of both the blade lean and the spanwise variation of circulation.

So, there are a bunch of differences from the actual turbine and its bi-dimensional midspan representation, some of which tend to improve its efficiency and some others to reduce it. On the contrary, in an H-type VAWT, the main substantial differences when shifting to a real 3D geometry are the presence of the struts (with the related surface friction drag) and of the tip blade effects due to the blade finite length (with the related induced drag). Both these differences act to lower the turbine efficiency, especially at high TSR, since the energy lost owing to these phenomena is roughly proportional to the square of the peripheral speed. Note in fact that the $2 \mathrm{D}$ simulations, opposite to the $3 \mathrm{D}$ ones, predict a greater maximum $C_{p}$ for the $\mathrm{H}$-shaped turbine (roughly 0.37 versus 0.34 ). As a final comment about this turbine-shape comparison, we can state that in a troposkein turbine what really matters is the flow field close the midspan section, where most of the mass is flowing. It is also for this reason that $2 \mathrm{D}$ and $3 \mathrm{D}$ outcomes of global parameters are not so far from each other, thus suggesting that a proper definition of the turbine solidity should refer to the equatorial section only.

\section{3D time-averaged flow field}

In order to better understand the troposkein turbine fluid-dynamics, its mean flow field was computed averaging 120 instantaneous data fields saved every 3 degrees during the last revolution. Since the inner part of the domain is rotating and thus its cell centers and nodes change their positions with time, the computational mesh at the beginning of the last lap $\left(\vartheta=0^{\circ}\right)$ was arbitrarily chosen as the reference one. Iso-contours of the most relevant fluid-dynamic quantities were plotted in 27 planes orthogonal to the cartesian coordinates (nine along each direction). The 27 surfaces are located at $X / R_{e q}=-1.0,-0.5,0.0,0.5,1.0,1.2,1.4,1.7$ (experimental plane) and 2.0 in the streamwise direction, at $Y / R_{e q}=-1.1,-0.9,-0.6,-0.3,0.0,0.3,0.6,0.9$ and 1.1 in the transverse direction, and at $Z / H^{*}=0.0$ (midspan), $0.25,0.5,0.625,0.75,0.875,1.0,1.15$ and 1.25 in the axial direction.

Note that when, for a particular angular position $\vartheta$, the chosen plane cuts a blade, the postprocessing software interpolates in the empty space occupied by the solid material the values assigned at its boundary. Hence, the velocities in the $\mathrm{X}$ and $\mathrm{Y}$ directions may appear perturbed inside the toroid generated by the blade rotation.

Looking at the time-averaged streamwise velocity in the equatorial plane, depicted in the left part of Figure 6, it is evident how it markedly differs from its bi-dimensional counterpart, shown in the right part of the same figure. The turbine wake at midspan remains confined and is characterized by a very small spreading angle. This sharply contrasts with its bi-dimensional behaviour and is due to the lower blockage exerted. The incoming flow, slowed down by the turbine, is not forced to bypass laterally the rotor, but can also slide axially where there is more free space. The same result is also found by other authors; for example, it can be appreciated in Figure 9 of [6]. This is not the case to occur in an H-shaped VAWT, unless the $H / D$ ratio is unreasonably small; the 2D and 3D midspan velocity fields for the H-VAWT investigated in [10] were indeed quite similar, and the related 2D and 3D wakes profiles at midspan had the same width.

Moving toward the tip of the troposkein blade, one can observe the same feature (see the left part of Figure 7) until the simultaneous great varations in TSR and $\sigma$, joined with the effect of friction at the blade insertion surface, completely alter the flow field. In the $Z 3$ plane, located halfway between midpsan and turbine top, the asymmetry between the windward $(Y>0)$ and 

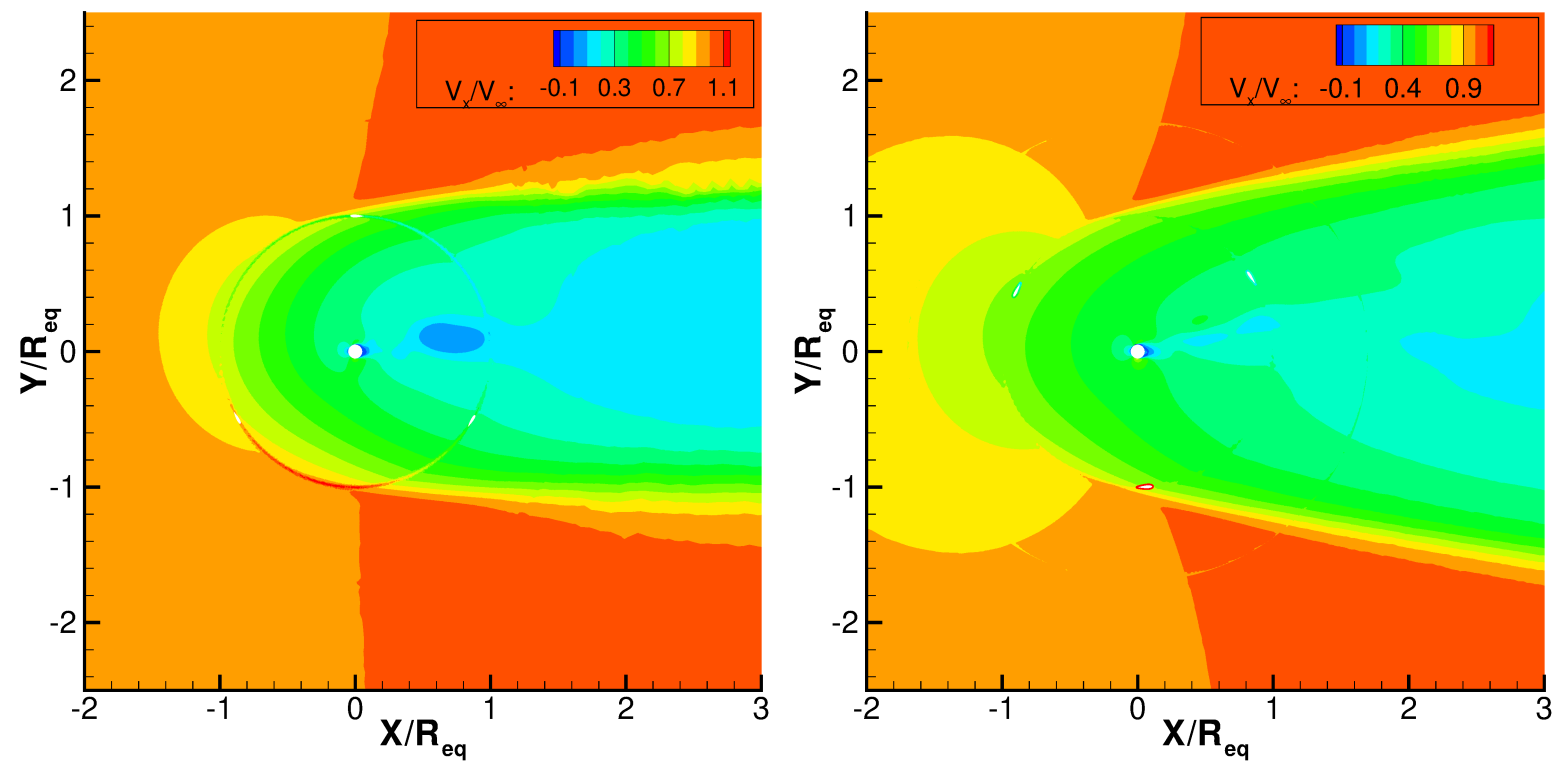

Figure 6: Non-dimensional streamwise velocity at $z / H^{*}=0(3 \mathrm{D})$ and in the $2 \mathrm{D}$ case.
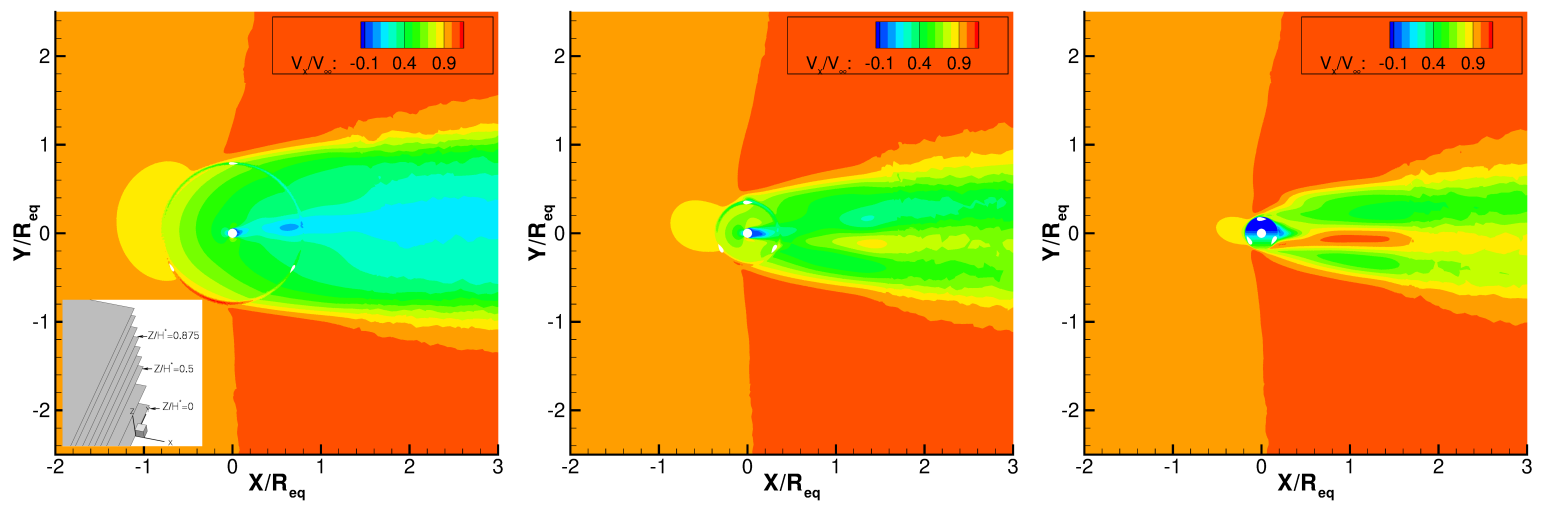

Figure 7: Non-dimensional streamwise velocity at $z / H^{*}=0.5$ (left), $z / H^{*}=0.875$ (center) and $z / H^{*}=1.0$ (right). 

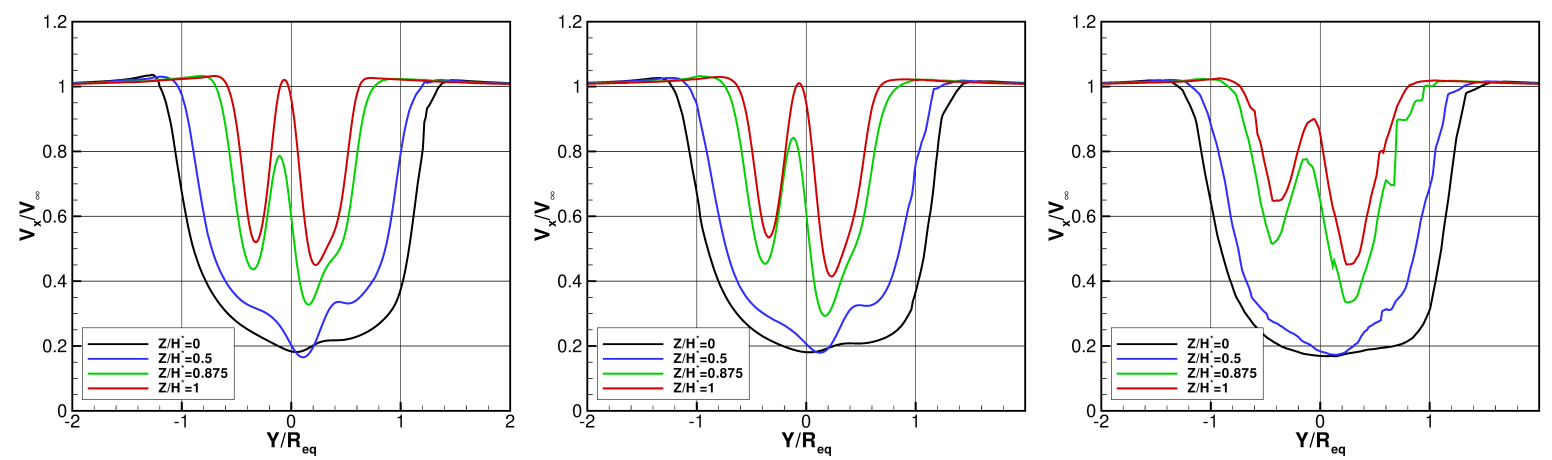

Figure 8: Non-dimensional streamwise velocity at $X / R_{e q}=1.2$ (left), $X / R_{e q}=1.4$ (center) and $X / R_{e q}=1.7$ (right), on four selected $z / H^{*}$ planes.
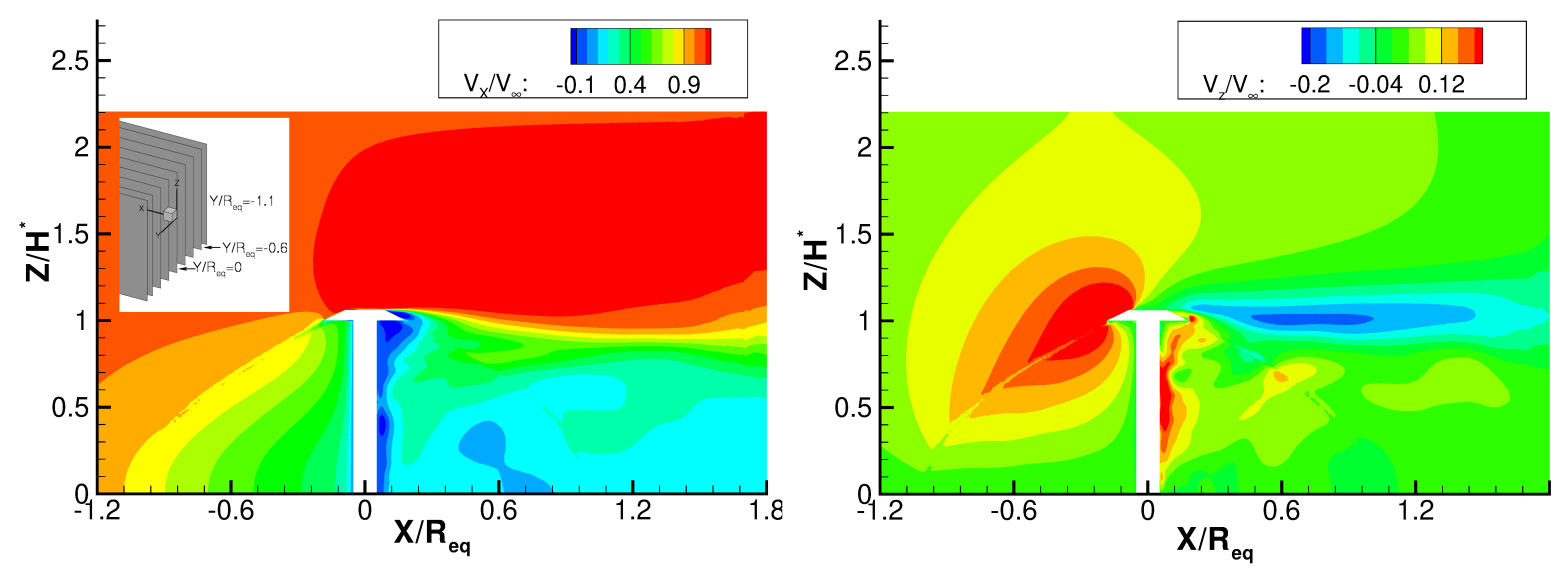

Figure 9: Non-dimensional streamwise (left) and axial (right) velocities at $Y / R_{e q}=0$.

the leeward $(Y<0)$ part of the wake starts to appear, becoming fully evident in the center part of Figure $7\left(Z / H^{*}=0.875\right)$. The local TSR has become small enough to induce periodic flow detachment (dynamic stall) in the leeward part of the revolution, impairing the extraction of work from the wind stream. Thus, there are greater velocities in the y-negative side of the wake. In the right part of the same figure, which shows the tip blade plane, another striking feature is visible: the flow acceleration behind the rotor near the $Y 5$ plane $(Y=0$.). This has to be ascribed to a 3D non-viscous phenomenon; namely the flow which goes down after having passed over the top the turbine. Further ensight into the turbine wake evolution is provided in Figure 8 where the streamwise velocity defect of four selected $z / H^{*}$ planes is illustrated at different streamwise positions (i.e. $\left.X / R_{e q}=1.2,1.4,1.7\right)$. These images further show how, moving downtream, the wake asymmetry becomes more and more evident for spanwise positions approaching the top where the local TSR value becomes smaller and smaller.

Figure 9 clearly illustrates the downwash after the turbine shaft and the re-energization of the tip wake flow in the region of negative axial velocity. As already mentioned, near the top of the turbine the blade-mast geometrical obstruction altogether with the surface friction and the huge solidity induce a dramatic blockage and the flow is forced to circumvent the rotor as if it were a solid. In front of the rotor (Figure 10), the spanwise displacement of the flow is already 

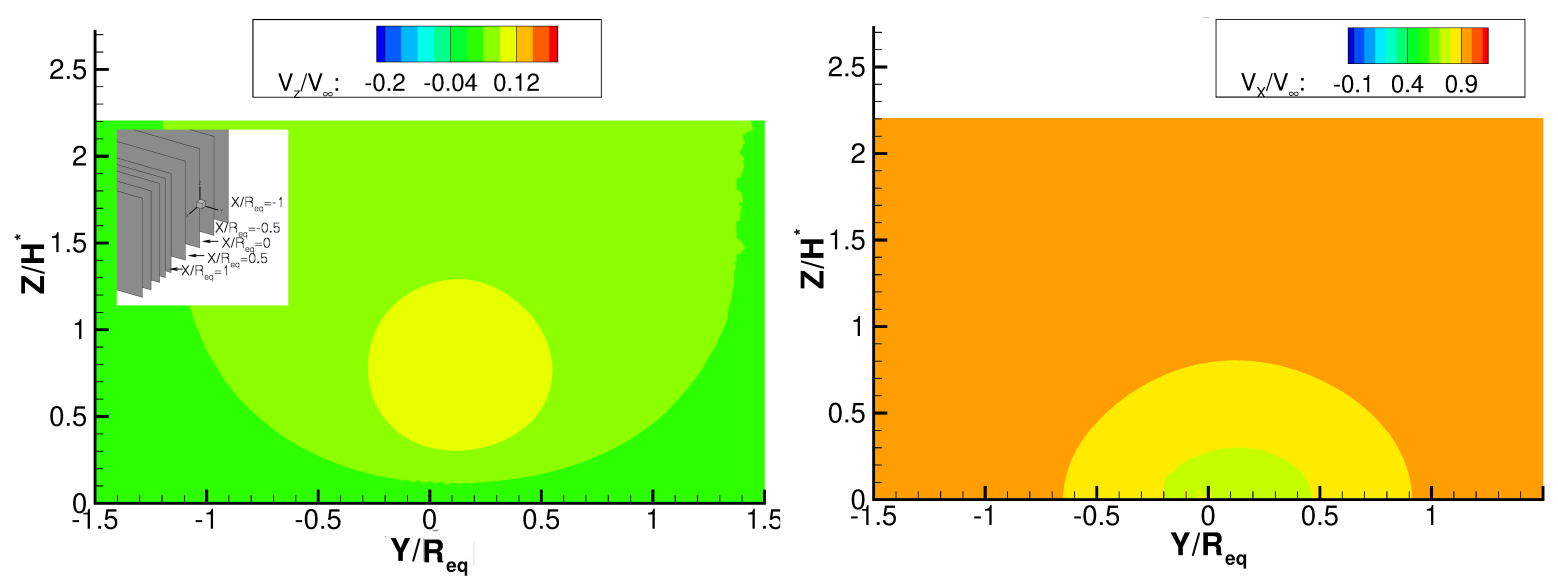

Figure 10: Non-dimensional spanwise (left) and streamwise (right) velocity at $X / R_{e q}=-1$.
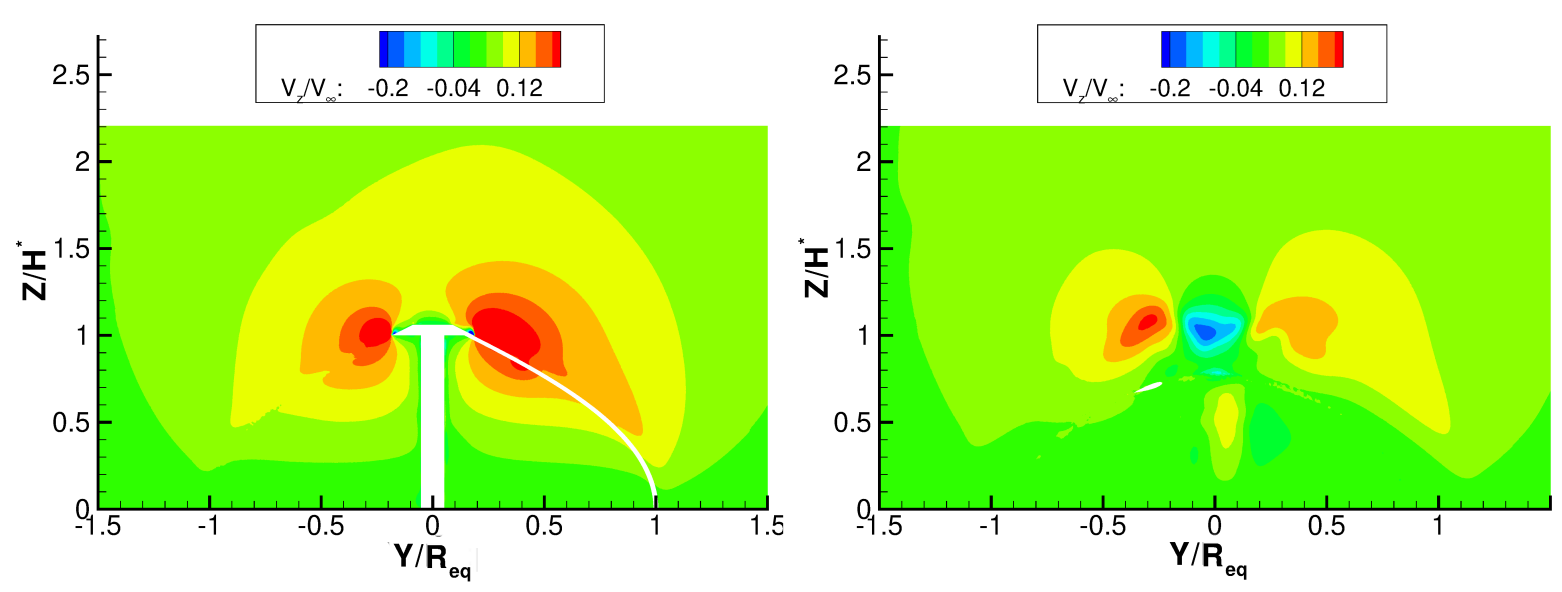

Figure 11: Non-dimensional spanwise velocity at $X / R_{e q}=0$. (left) and at $X / R_{e q}=0.5$ (right).

obvious, as well as its bending toward the leeward side, where there is minor resistance.

Figure 11 shows the iso-contours of the axial velocity in two x-constant planes: the first one cuts the axis of rotation and the second one is located afterwards, inside the rotor area. It is important to point out that, in the left picture, the axial velocity is greater going toward the tip not only because of the aforementioned increased blockage, but also because of the blade lean. In the upwind part of the revolution the lean acts to push the incoming stream upward, as the blade pressure side is the external surface; on the contrary in the downstream part of the revolution the blade pressure side is the internal surface and it pushes the flow downward. This last effect is of course more intense in the windward side, where the flow, even if slower that upwind, is less perturbed that in the leeward side by the possible boundary layer separation. It is in fact well evident, in the right picture, how the upward motion persists longer in the leeward side. Moreover, the downwash near the mid-longitudinal plane combines at the blade tip with this upward motion, creating two counter-rotating vortices, again well visible in the right part of Figure 11. Last but not least, the central part of the wake experiences an axial motion in the positive direction under the entrainment action of the local streamwise acceleration, motion also detected in the donwstream planes not shown here for the sake of brevity. 

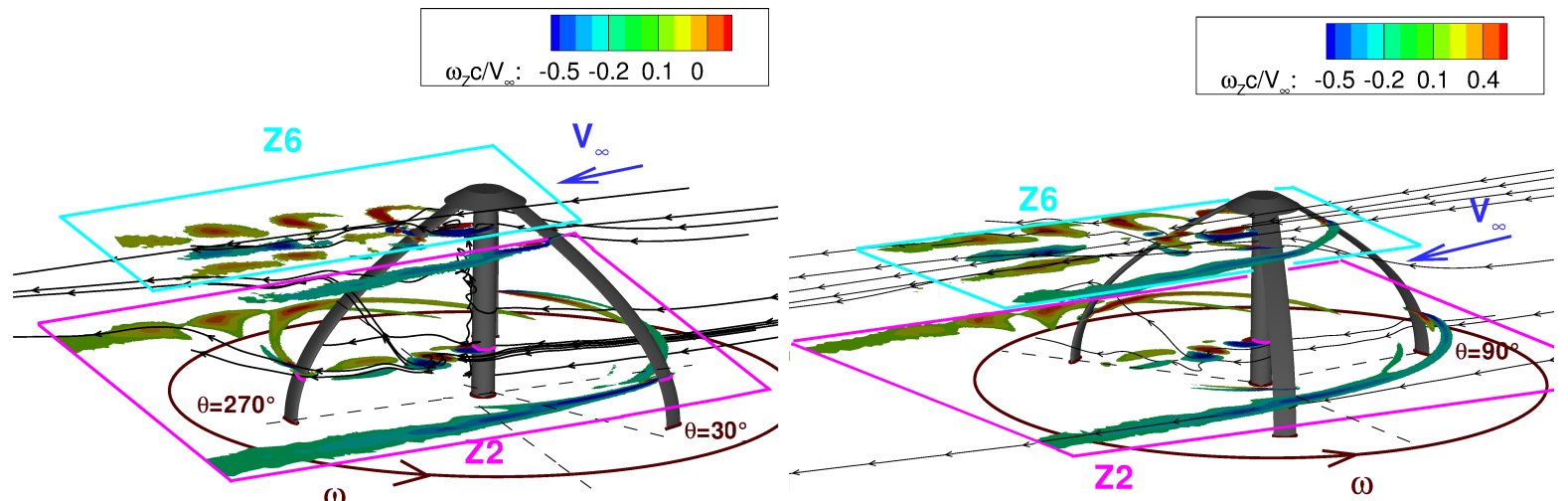

Figure 12: Non-dimensional axial vorticity for $\vartheta=30^{\circ}$ and $\vartheta=90^{\circ}$.
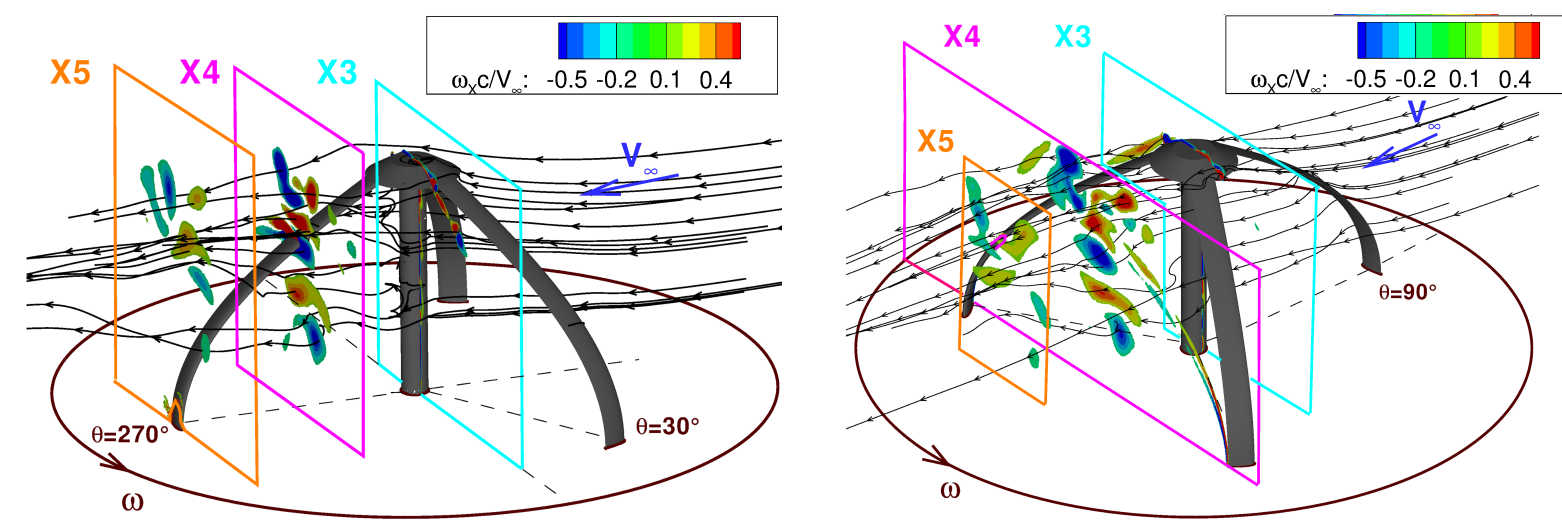

Figure 13: Non-dimensional streamwise vorticity for $\vartheta=30^{\circ}$ and $\vartheta=90^{\circ}$.

\section{3D instantaneous flow field}

This section briefly analyzes the instantaneous behaviour of the troposkein turbine for two selected angular positions of the rotor, when the azimuthal angle $\vartheta$ is $30^{\circ}$ and $90^{\circ}$. Contours of the normal component of vorticity are shown in planes $Z 2, Z 6$ and in planes $X 3, X 4, X 5$, together with some streamlines that give an idea of the fluid motion. Note that in these figures every plane is delimited by coloured solid lines only for the sake of clarity, to better fix which plane a countour belongs to. Streamlines instead are drawn in the full volume. Figure 12 shows the planes located at $z / H^{*}=0.25$ and 0.875 , whilst Figure 13 those at $X / R_{e q}=0 ., 0.5$, and 1 .

From the observation of Figure 12, one can notice that in the windward side only the trace of the turbine wake shear layer appears, whereas in the leeward side (and behind the mast) detached eddies of both positive and negative vorticity are transported by the flow. These structures are stronger close to the blade tip than near midspan, coherently with the lower local TSR approaching the top of the rotor. Streamlines highlight that the fluid in dead water region behind the shaft tends to be displaced axially outward. We also note that the axial vorticity in the blade boundary layer, during the upwind part of the revolution, is negative on the pressure (external) side and positive on suction (internal) one, the opposite occurring in the downwind part. 
Even more interesting is Figure 13. The streamwise vorticity of the blade boundary layer (remember that the profile is inclined in the axial-radial plane) has the same sign of its axial counterpart and, because of both blade lean and the spanwise variation of the load that induces a secondary vortical motion, it is shifted upward on the pressure side and downward on the suction side. Therefore positive and negative $x$-vorticity cores form after the passing blade, mutually interposed along the blade profile line (see plane $X 3$, especially in the left figure). In the snapshot taken when $\vartheta=90^{\circ}$ a positive core of streamwise vorticity is present near the tip on the leeward side, remaining of a blade passage. Observe that the vortices released behind the mast are well visible for all $\vartheta$ angular positions, while those shed by the blade only if the cutting plane is (afterward) not too far from the blade itself. From the same figure, one can also appreciate how on the leeward side the vortices are more pronounced. This is particularly evident approaching the top of the cited figures, since the local TSR becomes smaller and smaller and flow separations take place.

As a general comment, we have just seen that the vortices developing at a certain instant of time may well be completely different, in position, direction and intensity, from those present in the time-averaged flow field and a local and instantaneous flow analysis is mandatory if one wants to understand the details of the troposkein-VAWT aerodynamics.

\section{Concluding remarks}

This paper has presented a computational investigation on the flow around a troposkein VAWT, supported by a systematic experimental assessment based on wind-tunnel measurements. The focus of the paper has been on both the overall turbine performance and, for one operating condition, on the detailed three-dimensional development of the near wake. The results have been illuminating on several aspects that make the aerodynamics of this rotor different from that of the more standard, and most studied, H-shape one.

At first, 2D simulations on the equatorial plane, spanning over the whole operating range of the machine, have been shown to capture very well the experimental trend and performance data, despite the fully $3 \mathrm{D}$ character of the troposkein rotor. Several aspects contribute to alter the rotor aerodynamics in spanwise direction, some advantageous and some other disadvantageous in terms of performance; this complex combination ends up in a relatively limited impact of 3D effects on the performance (at least for the tested conditions). In fact, differently from the $\mathrm{H}$-shape turbine, most of the power harvested by the troposkein rotor is converted in the equatorial section, and also for this reason the $3 \mathrm{D}$ performance is close to the $2 \mathrm{D}$ one; as a further proof of that, the solidity evaluated on the equatorial section only has been found to be better representative of the turbine aerodynamics, with respect to a definition including the spanwise features of the blade.

Such a 2D-3D correspondence has not at all been found in terms of wake development. At first, the 2D simulation does not capture the experimental wake width even on the equatorial section, while the fully 3D model exhibits a very good agreement in terms of velocity distribution. Such a result, which contrasts with what found by the authors in previous works on a comparative $\mathrm{H}$-shape turbine, indicates that the $3 \mathrm{D}$ character of the rotor alters completely the development of the wake. The observed differences are probably due to the much reduced blockage that the rotor induces to the flow away from midspan; in these latter regions, interesting aerodynamic features appear. In time-averaged terms, the leaned shape of the blades of the troposkein rotor leads to the onset of two counter-rotating vortices, which ultimately generates a downwash of the flow in the central region of the near wake. Instantaneous pictures of the vorticity field indicate the shedding of intermittent vortex cores, featuring both streamwise 
and axial vorticity, marking the noteworthy complexity of the troposkein rotor aerodynamics. However, such vortices remain localized and their magnitude is not comparable with the tip vortex featuring comparative $\mathrm{H}$-shape rotors. Future works will search for confirmation of the observed features and effects for other operating conditions.

\section{Acknowledgements}

This work has been performed in the frame of the national ISCRA HPC grant T-WINDS (HP10CSUW23) within which the CINECA consortium provided the HPC facilities.

\section{References}

[1] F. Balduzzi, A. Bianchini, R. Maleci, G. Ferrara, and L. Ferrari. Critical issues in the CFD simulations of Darrieus wind turbines. Energy, 85:419-435, 2016.

[2] L. Battisti, E. Benini, A. Brighenti, S. Dell'Anna, M. Raciti Castelli, V. Dossena, G. Persico, U.S. Paulsen, and T.F. Pedersen. Wind Tunnel Testing of the DeepWind Demonstrator in Design and Tilted Operating Conditions. Energy, 111:484-497, 2016.

[3] L. Battisti, G. Persico, V. Dossena, B. Paradiso, M.R. Castelli, A. Brighenti, and E. Benini. Experimental benchmark data for h-shaped and troposkien VAWT architectures. Renewable Energy, 125:425-444, 2018.

[4] A. Bianchini, F. Balduzzi, G. Ferrara, G. Persico, V. Dossena, and L. Ferrari. A Critical Analysis on Low-Order Simulation Models for Darrieus VAWTs: How Much Do They Pertain to the Real Flow? Journal of Engineering for Gas Turbines and Power, 2019.

[5] L. Chao, Z. Sonye, X. You-Lin, and X. Yiqing. 2.5D large eddy simulation of vertical axis wind turbine in consideration of high angle of attack. Renewable energy, 51:317-330, 2013.

[6] P.L. Delafin, T. Nishino, A. Kolios, and N. Wang. Comparison of low-order aerodynamic models and rans cfd for full scale 3d vertical axis wind turbines. Renewable Energy, 109:564-575, 2017.

[7] U. Divakaran, V. Ratna Kishore, and A. Ramesh. Effect of wind speed on the performance of a troposkein vertical axis wind turbine. International J. of Renewable energy research, 109, N. 3, 2019.

[8] M. Elkhoury, T. Kiwata, and E. Aoun. Experimental and numerical investigation of a three-dimensional vertical-axis wind turbine with variable-pitch. J. Wind Eng. Ind. Aerodyn, 139:111-123, 2015.

[9] Carlos Simao Ferreira. The near wake of the VAWT, 2D and 3D views of the VAWT aerodynamics. PhD thesis, Delft University of Technology, Delft, the Nederlands, 2009.

[10] N. Franchina, G. Persico, and M. Savini. 2D-3D computations of a vertical axis wind turbine flow field: modeling issues and physical interpretations. Renewable Energy, 136:1170-1189, 2019.

[11] N. Franchina, G. Persico, and M. Savini. Three-dimensional unsteady aerodynamics of a H-shaped vertical axis wind turbine over the full operating range. Journal of Wind Engineering \& Industrial Aerodynamics, 206, 2020.

[12] H. F. Lam and H. Y. Peng. Study of wake characteristics of a vertical axis wind turbine by two- and three- dimensional computational fluid dynamics simulations. Renewable Energy, 90:386-398, 2016. 
[13] Q. Li, T. Maeda, Y. Kamada, J. Murata, M. Yamamoto, T. Ogasawara, K. Shizimu, and T. Kogaki. Study on power performance for a straight-bladed vertical axis wind turbine by field and wind tunnel test. Renewable Energy, 90:291-300, 2016.

[14] E. Mercker and J. Wiedemann. On the correction of the inter-ference effects in open jet wind tunnels. In SAE Technical paper, number 960671, 1996.

[15] A. Orlandi, M. Collu, S. Zanforlin, and A. Shires. 3D URANS analysis of a vertical axis wind turbine in skewed flows. Journal of Wind Engineering and Industrial Aerodynamics, 147:77-84, 2015.

[16] A. Ortolani, G. Persico, J. Drofelnik, A. Jackson, and M.S. Campobasso. Cross-comparative analysis of loads and power of pitching floating offshore wind turbine rotors using frequency-domain navier-stokes CFD and blade element momentum theory. Journal of Physics: Conference Series, 1618:052016, sep 2020 .

[17] I. Paraschivoiu. Wind Turbine Design: With Emphasis on Darrieus Concept. Polytechnic International Press, 2002. ISBN 9782553009310.

[18] G. Persico, V. Dossena, B. Paradiso, L. Battisti, A. Brighenti, and E. Benini. Time-resolved Experimental Characterization of Wakes Shed by H-Shaped and Troposkien Vertical Axis Wind Turbines. ASME Journal of Energy Resources Technology, 139(031203), Sept. 2017.

[19] A. Rezaeiha, I. Kalkamn, and B. Blocken. CFD simulation of a vertical axis wind turbine operating at a moderate tip speed ratio: guidelines for minimum domain size and azimuthal increment. Renewable Energy, 107:373-385, 2017.

[20] J. Saverin, G. Persico, D. Marten, D. Holst, G. Pechlivanoglou, and V. Dossena C. Paschereit. Comparison of experimental and numerically predicted three-dimensional wake behaviour of a vertical axis wind turbine. Journal of engineering for gas turbines and power, 140(122601), 2018.

[21] S. Shamsoddin and F. Porté-Agel. Large eddy simulation of vertical axis wind turbine wakes. Energies, 7:890-912, 2014.

[22] G. Tescione, D. Ragni, He C, S. Ferreira, and G.J.W. van Bussel. Near wake flow analysis of a vertical axis wind turbine by stereoscopic particle image velocimetry. Renewable Energy, 70:47-61, 2014.

[23] A. Untariou, H.G. Wood, P.E. Allaire, and R.J. Ribando. Investigation of self-starting capability of vertical axis wind turbines using a computational fluid dynamics approach. Journal of Solar Energy Engineering - ASME, 133:041010-1-8, 2011.

[24] Luca Vita, Uwe Schmidt Paulsen, Troels Friis Pedersen, Helge Aagaard Madsen, and Flemming Rasmussen. Deep wind: A novel floating wind turbine concept. Windtech International, 6(4):29-31, 2010. 